\title{
Design and analysis of a water pipe leakage sensor
}

\begin{abstract}
Water pipeline leakage is a common problem in almost every country in the world which has become a shared concern today. While some regions do not even have the access to clean and treated water, others have seen millions of litres of water wasted every day due to leakages which probably could have been sufficient to serve the needy. In most cases, the detrimental effects associated with the occurrence of leaks may present serious problems and therefore, leaks must be quickly detected, located and repaired. Recent advances in sensor technology have resulted in a wide application of sensor networks for the purpose of leakage management. Currently, researchers have gone as far as putting the sensors inside the pipeline itself to identify, locate and estimate the leak size. In the current study, CFD simulations were used to find the drag coefficient associated with the designs prepared which is an important parameter in this study. The work has been validated with the previous work. The main outcome from the study was the drag coefficient produced from the proposed model is significantly higher than the reference design. For the water pipe case, this a favourable outcome.
\end{abstract}

Keyword: Pipeline leakage; Leak detector; Water leakage simulation 